\title{
CORRELATION BETWEEN API 50 CH AND MULTIPLEX POLYMERASE CHAIN REACTION FOR THE IDENTIFICATION OF VAGINAL LACTOBACILLI IN ISOLATES
}

\author{
Eliane Melo Brolazo ${ }^{*}$, Domingos Silva Leite ${ }^{2}$, Monique Ribeiro Tiba ${ }^{2}$, Marina Villarroel ${ }^{1}$, Camila Marconi ${ }^{3}$, Jose Antonio \\ Simoes ${ }^{1}$
}

${ }^{1}$ Departamento de Obstetrícia e Ginecologia, Faculdade de Ciências Médicas, Universidade Estadual de Campinas, Campinas, SP, Brasil; ${ }^{2}$ Departamento de Microbiologia e Imunologia, Universidade Estadual de Campinas, Campinas, SP, Brasil; ${ }^{3}$ Departamento de Patologia, Faculdade de Medicina de Botucatu, Universidade Estadual Paulista, Botucatu, SP, Brasil.

Submitted: August 31, 2009; Returned to authors for corrections: October 16, 2009; Approved: November 04, 2010.

\begin{abstract}
Identification of Lactobacillus sp. strains by phenotypic methods may lead to doubtful results possibly interfering in the reliability of the epidemiological and probiotics studies. Therefore this study aimed to determine the best methodology for the identification of the large diversity of lactobacilli species found in the vagina by comparing two techniques, one based on their biochemical profile and other employing molecular biology. A carbohydrate fermentation test (API $50 \mathrm{CH}$ ) was compared with multiplex polymerase chain reaction (PCR) for the identification of species of vaginal lactobacilli from 135 healthy women. The kappa index was used to evaluate agreement between the methods. Using the molecular technique, $L$. crispatus $(32.6 \%)$, L. jensenii $(25 \%)$ and L. gasseri $(20.6 \%)$ were the most frequent species. However, using the biochemical technique, the most frequent species were: L. acidophilus (34.8\%), L. crispatus (27.2\%) and L. fermentum (13\%). Although L. acidophilus was the most frequent specie found by biochemical tests, no strain of this microorganism was detected by PCR. Agreement between the methods was low for identification of all the most common species. Although rates of L. crispatus detected were similar using both methods $(32.6 \%$ and $27.2 \%$ ), agreement between them was relatively low (kappa $=0.52)$. Conclusions: Our results confirmed the limitation of the biochemical method and the applicability of a previously published molecular method (Multiplex PCR) for the identification of lactobacilli in the vaginal tract, focusing on further necessity of its improvement for also targeting L. vaginalis and L. iners.
\end{abstract}

Key words: carbohydrate fermentation test; multiplex PCR; identification; vaginal lactobacilli; vaginal ecosystem

\section{INTRODUCTION}

Vaginal microflora of healthy women is normally composed by a large variety of aerobic and anaerobic bacteria.
However, in normal flora lactobacilli species, also known as Doderlein's bacillus, are predominant and have a significant effect on vaginal microbiota (17). These microorganisms inhibit the growth of potential pathogens by competing for

\footnotetext{
*Corresponding Author. Mailing address: Caixa Postal 6181 - cep 13084-971, Campinas, SP, Brazil.; Tel.: +55-19-3289-2856 Fax: +55-19-3289-2440.; E-
} mail: elianeb@unicamp.br 
space and nutrients $(3,16)$. Lactobacilli species also produce several antimicrobial substances such as organic acids, hydrogen peroxide $\left(\mathrm{H}_{2} \mathrm{O}_{2}\right)$ and bacteriocins (7). Organic acid release by lactobacilli maintains vaginal $\mathrm{pH} \leq 4.5$, creating an inhospitable environment for pathogens. In addition to acid production, the combination of hydrogen peroxide $\left(\mathrm{H}_{2} \mathrm{O}_{2}\right)$ and bacteriocins suppresses the endogenous pathogenic flora and maintains the local equilibrium (5). There are inherent differences among the vaginal lactobacilli isolates regarding their ability of conferring local protection. Since some species are known to be predominant in healthy women, presumably those are the most capable of inhibiting pathogenic bacteria. Therefore, much importance resides on the correct taxonomic identification of lactobacilli species for the development of epidemiological studies and also to enable the use of vaginal lactobacilli as probiotics (2).

Taxonomic identification by manufactured kits such as API $50 \mathrm{CH}$ (bioMerieux, Craponne France), which are based on phenotypical characteristics are still widely used. $L$. acidophilus is the most common species of vaginal lactobacilli identified by this method (1). However phenotypic methods may identify lactobacilli belonging to different species as the same microorganism, which explains the variability reported by epidemiological studies performed using this methodology for lactobacilli identification (15). Currently, the definition of Lactobacillus species is based on the fermentative profile (phenotype), albeit practical, is known to be an inaccurate method for identification (11).

Recently, the use of genetic analyses represented an advance in the taxonomy of lactobacilli. Studies based on the DNA homology of lactobacilli concluded that some strains previously classified according to their phenotype as $L$. acidophilus, in fact consists of six different groups that cannot be differentiated biochemically. These groups were then genetically classified into six different species: L. acidophilus, L. crispatus, L. amylovorus, L.gallinarum, L. gasseri and L. jensenii (11). Later studies carried out using molecular biology revealed that $L$. crispatus, L. gasseri and L. jensenii are the most common species in vaginal environment (13).

Although identification based on DNA homology allowed better knowledge on the taxonomic relationships among lactobacilli species, the identification of vaginal isolates by this method is extremely laborious. Therefore, faster and more reliable methods need to be developed to determine the distribution of vaginal lactobacilli species (14).

There are several methods of molecular biology tools that are used for bacterial identification, such as randomly amplified polymorphic DNA (RAPD) (16), amplified ribosomal DNA restriction analysis (ARDRA), ribotyping and subsequent comparison with the ribosomal database project (RDP) (9), and sequencing and comparison with GenBank databases (www.ncbi.nlm.nih.gov) (18) or even the amplification of regions of bacterial DNA using multiplex PCR with primers that produce fragments of DNA of different sizes for each species (14).

Multiplex PCR technique uses primers that are designed based on specific sequences of bacterial DNA that codify the regions of the $16 \mathrm{~S}$ and $23 \mathrm{~S}$ ribosomal RNA, which are exclusive for each species and have been used successfully in the identification of intestinal lactobacilli (14). Thus, the objective of this study was to compare the identification of lactobacilli isolated from vaginal samples of healthy women using two different methods, one based on the biochemical profile using API CH50 carbohydrate fermentation test and a second one based on the multiplex PCR technique developed by Song et al (2000) (14).

\section{MATERIAL AND METHODS}

The study was conducted at the Department of Obstetrics and Gynaecology and Women Hospital, School of Medical Sciences, University of Campinas (UNICAMP), Campinas, Brazil. A total of 135 women at reproductive age without gynecologic diseases or complaints of vaginal discharge were 
selected and included in the study. This study was approved by the local IRB, approval letter \#202/2005 and all women signed an inform consent prior to enrollment. During gynaecological examination, vaginal samples were collected for the isolation of lactobacilli and for Gram-stained vaginal smears for confirmation of the absence of vaginal infections. In the Gramstained smears, bacterial morphotypes were quantified in accordance with Nugent's criteria (10). Only women with scores from 0 to 3 were considered normal and thus included in the study.

The samples were collected in Amies charcoal transport medium and were seeded into two plates of selective medium (MRS Agar - Oxoid, Basingstoke, UK) and incubated at $37^{\circ} \mathrm{C}$ for 24-48 hours at anaerobic atmosphere (Forma Anaerobic System, Thermo Electron Corporation, Waltham, MA, USA)and at $5 \% \mathrm{CO}_{2}$ (Electron Series II Water-Jacketed $\mathrm{CO}_{2}$ incubator, Thermo Electron Corporation). The isolated colonies were preliminarily identified based on their morphological and staining characteristics (Gram-positive bacilli) and their catalase reaction (negative), and stored at $-70^{\circ} \mathrm{C}$ in MRS broth supplemented with glycerol until being submitted to the carbohydrate fermentation test using the API $50 \mathrm{CH}$ carbohydrate fermentation strips (bioMerieux, Craponne, France). The results were analyzed according to the biochemical profiles registered in the APIweb® database (bioMerieux).

For molecular identification, DNA from isolates was extracted and purified using the MasterPure gram-positive DNA purification kit and Ready-Lyse lysozyme solution (Epicentre Biotechnologies, Madison, WI, USA). Identification of the species was performed by multiplex polymerase chain reaction (PCR) in two stages as described by Song et al. (2000) (14). Because there are many possible Lactobacillus sp. that can be potentially indentified and there are similarities present in their genotypes, this method consisted in a two-step process in order to compose two groups previously to the final identification. A set of primers denominated multiplex PCR-G was used to group the lactobacilli. According to the size of the amplified fragment, another set of primers, denominated Multiplex PCR-G2A and G2B, Multiplex PCR-G3 and Multiplex PCR-G4, were used to identify the species in each one of the four groups. The target species of the Multiplex PCR were: L. delbrueckii, L. acidophilus, L. crispatus, L. gasseri, L. jensenii, L. paracasei, L. rhamnosus, L. fermentum, L. plantarum, L. reuteri and L. salivarius.

The reactions were carried out in a Mastercycler gradient thermocycler (Eppendorf AG, Hamburg, Germany) with a final volume of $30 \mu \mathrm{l}$ composed of $1.5 \mathrm{U}$ Taq DNA polymerase, $3 \mu \mathrm{l}$ of reaction buffer, $2.4 \mu \mathrm{l}$ of $\mathrm{MgCl}_{2}(25 \mathrm{mM}), 0.24 \mu \mathrm{l}$ of dCTP, dATP, dGTP and dTTP 25nM (Fermentas, Ontario, Canada), 1 $\mu \mathrm{l}$ of each primer $(30 \mathrm{ng} / \mu \mathrm{l})$ of the respective groups and $2 \mu \mathrm{l}$ of DNA template.

The specific primers were designed based on the DNA sequences that codify the intergenic spacer region between $16 \mathrm{~S}$ and 23S ribosomal RNA (Table 1). The amplification reactions were modified from the original publication as follows: reactions of 30 cycles that consisted of $94^{\circ} \mathrm{C}$ for 1 minute for denaturation; annealing at $60^{\circ} \mathrm{C}$ for 1 minute for multiplex PCR-G, $56^{\circ} \mathrm{C}$ for the others; followed by 1 minute at $72^{\circ} \mathrm{C}$ for extension. At the end of 30 cycles, 5 minutes at $72^{\circ} \mathrm{C}$ for final extension. The amplicons were analyzed by electrophoresis in $2.5 \%$ ethidium bromidestained agarose gel.

To standardize the method, 11 standard strains acquired from the American Type Culture Collection (ATCC) were used: L. delbrueckii (ATCC 10705), L. acidophilus (ATCC 4356), L. crispatus (ATCC 33820), L. gasseri (ATCC 9857), L. jensenii (ATCC 25258), L. paracasei (ATCC 27092), L. rhamnosus (ATCC 53103), L. fermentum (ATCC 14932), L. plantarum (ATCC 14917), L. reuteri (ATCC 5 53608) and $L$. salivarius (ATCC 29602).

To statistical analysis the kappa index was used to assess the comparison between the two methods and agreements were classified as follows: excellent (1.00- 0.80), good (0.79-0.60), moderate $(0.59-0.40)$, fair $(0.39-0.20)$, poor $(0.19-0.00)$ or in 
total disagreement $(<0.0)(12)$.

Table 1. Sequences of oligonucleotides used in the PCRs for identification of lactobacilli in each step and corresponding species and $\operatorname{size}^{14}$.

\begin{tabular}{|c|c|c|c|c|}
\hline First step & Name & Sequence (5'-3') & Species & Product \\
\hline \multirow[t]{2}{*}{ Group I } & Ldel-7 & ACAGATGGATGGAGAGCAGA & L. delbrueckii & $450 \mathrm{bp}$ \\
\hline & Lac-2 & CCTCTTCGCTCGCCGCTACT & & \\
\hline \multirow[t]{2}{*}{ Group II } & LU-1P & ATTGTAGAGCGACCGAGAAG & Go to PCR 1 & $300 \mathrm{bp}$ \\
\hline & Lac-2 & CCTCTTCGCTCGCCGCTACT & & \\
\hline \multirow[t]{2}{*}{ Group III } & LU-5 & CTAGCGGGTGCGACTTTGTT & Go to PCR 2 & $400 \mathrm{bp}$ \\
\hline & Lac-2 & CCTCTTCGCTCGCCGCTACT & & \\
\hline \multirow[t]{2}{*}{ Group IV } & LU-3P & AAACCGAGAACACCGCGTT & Go to PCR 3 & $350 \mathrm{bp}$ \\
\hline & Lac-2 & CCTCTTCGCTCGCCGCTACT & & \\
\hline \multicolumn{5}{|l|}{ Second step } \\
\hline \multirow[t]{3}{*}{ PCR 1} & Laci-1 & TGCAAAGTGGTAGCGTAAGC & Lacidophilus & $210 \mathrm{bp}$ \\
\hline & Ljen-3 & AAGAAGGCACTGAGTACGGA & L. jensenii & $700 \mathrm{bp}$ \\
\hline & $23-10 \mathrm{C}$ & CCTTTCCCTCACGGTACTG & & \\
\hline \multirow[t]{4}{*}{ PCR 2} & Lcri-3 & AGGATATGGAGAGCAGGAAT & L. cripatus & $522 \mathrm{bp}$ \\
\hline & Lcri-2 & CAACTATCTCTTACACTGCC & & \\
\hline & Lgas-3 & AGCGACCGAGAAGAGAGAGA & L. gasseri & $360 \mathrm{bp}$ \\
\hline & Lgas-2 & TGCTATCGCTTCAAGTGCTT & & \\
\hline \multirow[t]{3}{*}{ PCR 3} & Lpar-4 & GGCCAGCTATGTATTCACTGA & L. paracasei & $312 b p$ \\
\hline & RhaII & GCGATGCGAATTTCTATTATT & L. rhamnosus & $113 b p$ \\
\hline & LU-5 & CTAGCGGGTGCGACTTTGTT & & \\
\hline \multirow[t]{8}{*}{ PCR 4} & Lfer-3 & ACTAACTTGACTGATCTACGA & L. fermentum & $192 \mathrm{bp}$ \\
\hline & Lfer-4 & TTCACTGCTCAAGTAATCATC & & \\
\hline & Lpla-3 & ATTCATAGTCTAGTTGGAGGT & L. plantarum & $248 \mathrm{bp}$ \\
\hline & Lpla-2 & CCTGAACTGAGAGAATTTGA & & \\
\hline & Lreu-1 & CAGACAATCTTTGATTGTTTAG & L. reuteri & $303 b p$ \\
\hline & Lreu-4 & GCTTGTTGGTTTGGGCTCTTC & & \\
\hline & Lsal-1 & AATCGCTAAACTCATAACCT & L. salivarius & $411 \mathrm{bp}$ \\
\hline & Lsal-2 & CACTCTCTTTGGCTAATCTT & & \\
\hline
\end{tabular}




\section{RESULTS}

Ninety-two strains of lactobacilli were isolated from the 135 vaginal samples. The results according to Multiplex PCR and API are shown in Table 2. In the identification by Multiplex PCR, L. crispatus (32.6\%) was the most common lactobacilli species in the vagina of healthy women, followed by L. jensenii (25\%) and L. gasseri (20.6\%). According to biochemical profiles obtained with the API test, the most frequently detected species were L. acidophilus (34.8\%), followed by L. crispatus (27.2\%) and L. fermentum (13\%). Although the most frequently identified by biochemical profile (API $50 \mathrm{CH}$ (bioMerieux, Craponne France) was $L$. acidophilus, we did not detect any strain of this microorganism by Multiplex PCR. Similarly, L. gasseri and L. jensenii were frequently identified by Multiplex PCR, but no strains of these species were found by the biochemical test.
Kappa index value was low for the majority of the detected species (Table 3). Although the frequency of $L$. crispatus was similar by both methods $(32.6 \%$ with PCR and $27.2 \%$ with API $50 \mathrm{CH}$ ), the agreement rate between them was low $($ kappa $=0.52)$. Agreement between API $50 \mathrm{CH}$ and Multiplex PCR for the identification of L. salivarius was $100 \%$ for the only case of this species identified among all the 92 species isolated in this study.

The 11 ATCC strains of lactobacilli that were used as standard controls in Multiplex PCR were also assessed by API $50 \mathrm{CH}$ test and there were four cases of misidentification using this method. According to the biochemical profile, L. jensenii (ATCC 252580) and L. gasseri (ATCC 9857) were identified as L. acidophilus, and L. reuteri (ATCC 53608) as $L$. fermentum, while identification of L. rhamnosus (ATCC 53103) was found to be inconclusive (data not shown).

Table 2. Lactobacillus species identified according to Multiplex PCR and the API identification software database.

\section{Identification based on Multiplex PCR}

\section{API 50 CH L. crispatus L.jensenii L.gasseri $\quad$ L. sp $\quad$ L. delbrueckii L. fermentum L. reuteri L. rhamnosus L. salivarius Total N (\%)}

\begin{tabular}{|c|c|c|c|c|c|c|c|c|c|c|}
\hline L. crispatus & 19 & 5 & 1 & - & - & - & - & - & - & $25(27.2 \%)$ \\
\hline L. jensenii & - & - & - & - & - & - & - & - & - & - \\
\hline L. $s p$ & - & - & - & - & - & - & - & - & - & - \\
\hline L. gasseri & - & - & - & - & - & - & - & - & - & - \\
\hline L. delbrueckii & 3 & - & 6 & - & - & - & - & - & - & $9(9.8 \%)$ \\
\hline L. fermentum & - & - & - & 7 & 1 & 2 & 2 & - & - & $12(13.0 \%)$ \\
\hline L. reuteri & - & - & - & - & - & - & - & - & - & - \\
\hline L. rhamnosus & - & - & - & - & 1 & - & - & 2 & - & $3(3.3 \%)$ \\
\hline L. salivarius & - & - & - & - & - & - & - & - & 1 & $1(1.1 \%)$ \\
\hline L. acidophilus & 6 & 13 & 12 & 1 & - & - & - & - & - & $32(34.8 \%)$ \\
\hline L. brevis & - & 1 & - & 2 & - & - & - & - & - & $3(3.3 \%)$ \\
\hline L. plantarum & 2 & 4 & - & - & - & - & - & - & - & $6(6.5 \%)$ \\
\hline L. paracasei & - & - & - & 1 & - & - & - & - & - & $1(1.1 \%)$ \\
\hline Total N (\%) & $30(32.6 \%)$ & $23(25 \%)$ & $19(20.6 \%)$ & $11(12 \%)$ & $2(2.2 \%)$ & $2(2.2 \%)$ & $2(2.2 \%)$ & $2(2.2 \%)$ & $1(1.1 \%)$ & \\
\hline
\end{tabular}


Table 3. Agreement in the diagnosis of lactobacilli by molecular and biochemical methods $(\mathrm{n}=92)$.

\begin{tabular}{|c|c|c|c|c|c|}
\hline \multicolumn{4}{|c|}{ In agreement } & \multicolumn{2}{|c|}{ Kappa (95\% CI) } \\
\hline & Negative & Positive & $\%$ of agreement & & \\
\hline L. crispatus & 56 & 19 & $(81.5)$ & 0.52 & $(0.32-0.71)$ \\
\hline L. delbrueckii & 81 & 0 & $(88.0)$ & -0.04 & $(-0.08-0.01)$ \\
\hline L. fermentum & 80 & 2 & $(89.1)$ & 0.26 & $(-0.03-0.55)$ \\
\hline L. gasseri & 73 & 0 & $(80.4)$ & NP & \\
\hline L. jensenii & 69 & 0 & $(78.3)$ & NP & \\
\hline L. reuteri & 91 & 0 & (98.9) & NP & \\
\hline L. salivarius & 91 & 1 & $(100.0)$ & 1.00 & $(1.00-1.00)$ \\
\hline L. $s p$ & 81 & 0 & $(88.0)$ & -0.04 & $(-0.08-0.01)$ \\
\hline L. rhamnosus & 89 & 2 & $(98.9)$ & NP & \\
\hline
\end{tabular}

$\mathrm{NP}=$ not possible to calculate

$95 \% \mathrm{CI}=95 \%$ confidence interval

\section{DISCUSSION}

Agreement between multiplex PCR and API $50 \mathrm{CH}$ was poor for the majority of the species. Agreement was $100 \%$ only for L. salivarius, which had only one single strain detected among all the evaluated species, while for $L$. crispatus agreement was $81.5 \%$. According to the biochemical profiles by API $50 \mathrm{CH}$ test applied to the APIweb® database system, the majority of vaginal lactobacilli isolates were identified as $L$. acidophilus (32.6\%), which was not detected by Multiplex PCR identification. The group of lactobacilli previously classified as L. acidophilus was genetically evaluated and reclassified as six different species, among them L. crispatus, L. gasseri, and L. jensenii, which were frequently isolated in the vagina of healthy women (13). According to PCR results, L. crispatus was the most frequently species detected, followed by $L$. jensenii and L. gasseri. These findings were in agreement to other studies in which molecular techniques were used to identify lactobacilli isolated from healthy women $(8,11)$. Neither L. jensenii nor L. gasseri, respectively the second and third most frequently found species in this study, were identified by API, because their biochemical profiles do not exist in the database of the APIweb® system (2). Other species (L. fermentum, L. delbrueckii, L. reuteri, L. rhamnosus and L. salivarius) are rarely found in the vagina of healthy women, suggesting that they have fewer competitive characteristics compared to the most frequent species.

Only two species were detected with similar rates by the two methods. One was L. crispatus with a frequency of $32.6 \%$ by Multiplex PCR and $27.2 \%$ by API $50 \mathrm{CH}$ test. Nevertheless, agreement was moderate, since only 19 strains were identified concomitantly by both methods as L. crispatus. L. salivarius was the only strain that had an agreement that was considered excellent (kappa=1.00); however, this result referred to the only strain of this species isolated in this study.

Even the reference strains used for the standardization of Multiplex PCR were incorrectly identified by the biochemical method. L. jensenii and L. gasseri were identified biochemically as L. acidophilus, which was already expected, since the biochemical profiles of these two species are not sufficiently different from the profile of L. acidophilus and are not even listed as identifiable species in this system. However, L. reuteri was identified as L. fermentum and identification of L. rhamnosus by APIweb® system was inconclusive. 
Other studies using molecular biology techniques have reported that, in addition to $L$. crispatus, $L$. jensenii, and $L$. gasseri, other frequently found species are $L$. iners and $L$. vaginalis (2). L. iners was firstly described by Falsen and coworkers (6) as being a Gram-positive, facultative anaerobic rod-shaped bacterium. However, L. iners differs from other lactobacilli since it cannot be cultivated in MRS medium and its morphological and staining characteristics vary widely. Moreover, this species was described as consisting of a short and relatively Gram-negative bacillus (4). Since Nugent's criteria was followed for women inclusion in this study, the possible presence of women with a prevalence of $L$. iners in their flora may have been interpreted as indicative of the absence of lactobacilli and therefore excluded from the study. In addition, MRS medium was used for the cultivation and isolation of the lactobacilli and this medium would not have permitted the growth of $L$. iners possibly present in our samples.

Among the $12 \%$ of strains unidentified by Multiplex PCR, there may be a predominant species (probably L. vaginalis) that was not identified because there is no specific group for this species in the set of primers used in this study, which was based on methodology developed to identify species of intestinal lactobacilli. Based on these results, we intend to sequence these strains of Lactobacillus sp in order to identify them and use the resulting data to design specific primers for inclusion in the Multiplex PCR identification technique for vaginal lactobacilli.

In conclusion, our results confirm that the use of biochemical methods does not appear to be appropriate for the identification and study of vaginal lactobacilli, since the failure rate with this method was high compared to molecular biology techniques. In addition, our results also confirmed the applicability of Multiplex PCR for the identification of lactobacilli in the vagina, focusing on further necessary improvement of that method, targeting to $L$. vaginalis and $L$. iners.

\section{ACKNOWLEDGMENTS}

This project was partially supported by the Fundacao de Amparo a Pesquisa do Estado de Sao Paulo (FAPESP), grant\# 05/52649-4. The authors gratefully acknowledge Sirlei Morais for collaborating in the statistical analysis.

\section{REFERENCES}

1. Boskey, E.R.; Telsch, K.M.; Whaley, K.J.; Moench, T.R.; Cone, R. A. (1999). Acid production by vaginal flora in vitro is consistent with the rate and extent of vaginal acidification. Infect. Immun. 67, 5170-5175.

2. Boyd, M.A.; Antonio, M.A.; Hillier, S. L. (2005). Comparison of 5 API $50 \mathrm{CH}$ strips to wholechromosomal DNA probes for identification of Lactobacillus species. J. Clin. Microbiol. 43, 5309-5311.

3. Chan, R.C.; Reid, G.; Irvin, R.T.; Bruce A.W.; Costerton J. W. (1985). Competitive exclusion of uropathogens from human uroepithelial cells by Lactobacillus whole cells and cell wall fragments. Infect Immun. 47, 84-89.

4. De Backer, E.; Verhelst, R.; Verstraelen, H.; Alqumber, M.A.; Burton J.P.; Tagg, J.R.; Temmerman, M.; Vaneechoutte M. (2007). Quantitative determination by real-time PCR of four vaginal Lactobacillus species, Gardnerella vaginalis and Atopobium vaginae indicates an inverse relationship between L. gasseri and L. iners. BMC Microbiol. 7, 115 .

5. Dembélé, T.; Obdrzalek, V.; Votava, M. (1998). Inhibition of bacterial pathogens by lactobacilli. Zentralbl. Bakteriol. 288, 395-401.

6. Falsen E.; Pascual C.; Sjoden B.; Ohlen M.; Collins M. D. (1999). Phenotypic and phylogenetic characterization of a novel Lactobacillus species from human sources: description of Lactobacillus iners sp. nov. Int. J. Syst. Bacteriol. 49, 217-221.

7. Kaewsrichan, J.; Peeyananjarassri, K.; Kongprasertkit J. (2006). Selection and identification of anaerobic lactobacilli producing inhibitory compounds against vaginal pathogens. FEMS Immunol. Med. Microbiol. $48,75-83$.

8. Kilic, A.O.; Pavlova, S.I.; Alpay, S.; Kilic, S.S.; Tao L. (2001). Comparative study of vaginal Lactobacillus phages isolated from women in the United States and Turkey: prevalence, morphology, host range, and DNA homology. Clin. Diagn. Lab. Immunol. 8, 31-39.

9. Maidak, B.; Cole, J.R.; Lilburn, T.G.; Parker. C.T. Jr.; Saxman, P.R.; Farris, R.J.; Garrity, G.M.; Olsen, G.J.; Schmidt, T.M.; Tiedje J.M. (2001). Nucleic Acids Res. 29, 173-174. The RDP-II (Ribosomal Database Project, release 10; http://rdp.cme.msu.edu).

10. Nugent, R.P.; Krohn M.A.; Hillier S. L. (1991). Reliability of diagnosing bacterial vaginosis is improved by a standardized method of Gram strain 
interpretation. J. Clin. Microbiol. 29, 297-301.

11. Pavlova, S.I.; Kilic, A.O.; Kilic, S.S.; So, J.S.; Nader-Macias; M.E., Simoes; J.A.; Tao L. (2002). Genetic diversity of vaginal lactobacilli from women in different countries based on 16S rRNA gene sequences. J. Appl. Microbiol. 92, 451-459.

12. Siegel, S.; Castellan N. (1998). Nonparametric Statistics for the Behavioral Sciences. McGraw-Hill, New York.

13. Song, Y.L.; Kato, N.; Matsumiya, Y.; Li, C.X.; Kato, H.; Watanabe K. (1999). Identification of and hydrogen peroxide production by fecal and vaginal lactobacilli isolated from Japanese women and newborn infants. J. Clin. Microbiol. 37, 3062-3064.

14. Song, Y.; Kato; N., Liu C.; Matsumiya, Y.; Kato, H.; Watanabe K. (2000). Rapid identification of 11 human intestinal Lactobacillus species by multiplex PCR assays using group and species-specific primers derived from the 16S-23S rRNA intergenic spacer region and its flanking 23S rRNA. FEMS Microbiol. Lett. 187, 167-173.
15. Vasquez, A.; Jakobsson, T.; Ahrne, S.; Forsum, U.; Mollin G. (2002). Vaginal Lactobacillus flora of healthy Swedish women. J. Clin. Microbiol. 40, 2746-2749.

16. Velraeds, M.M.; van der Mei, H.C.; Reid, G.; Busscher H. J. (1996). Inhibition of initial adhesion of uropathogenic Enterococcus faecalis by bio-surfactants from Lactobacillus isolates. Appl. Environ. Microbiol. 62, 1958-1963.

17. Wilks, M.; Wiggins, R.; Whiley, A.; Hennessy, E.; Warwich, S.; Porter, H.; Corfield, A.; Millar M. (2004). Identification and $\mathrm{H}_{2} \mathrm{O}_{2}$ production of vaginal lactobacilli 5 from pregnant women at high risk of preterm birth and relation with outcome. J. Clin. Microbiol., 42, 713-717.

18. Zhou, X.; Bent, S.J.; Schneider, M.G.; Davis, C.C.; Islam, M.R.; Forney L. J. (2004). Characterization of vaginal microbial communities in adult healthy women using cultivation-independent methods. Microbiology. 150, 2565-2573. 